\title{
Dissolution Media for In Vitro Testing of Water-Insoluble Drugs: Effect of Surfactant Purity and Electrolyte on In Vitro Dissolution of Carbamazepine in Aqueous Solutions of Sodium Lauryl Sulfate
}

\author{
John R. Crison ${ }^{\dagger x}$, Norman D. Weiner ${ }^{\ddagger}$, And Gordon L. Amidon ${ }^{\ddagger}$
}

Received February 29, 1996, from the ${ }^{\top} T S R L$, Inc., 540 Avis Drive, Ann Arbor, MI 48108, and the ${ }^{\ddagger}$ College of Pharmacy, The University of Michigan, 428 Church St. Ann Arbor, MI 48109-1065. publication October $9,1996^{\otimes}$.

Final revised manuscript received August 29, 1996.

Accepted for

\begin{abstract}
The intrinsic dissolution rate and solubility of carbamazepine was measured in aqueous solutions of sodium lauryl sulfate (SLS) prepared with two different grades of purity, 95 and $99 \%$, and $95 \%$ SLS in $0.15 \mathrm{M} \mathrm{NaCl}$ to determine the effect of surface-active impurities and electrolytes. Four significant observations resulted from this work: (1) the equilibrium coefficients calculated from the solubility experiments in the $99 \%$ SLS, $95 \%$ SLS, and $95 \%$ with $0.15 \mathrm{M} \mathrm{NaCl}$ SLS solutions were 295,265 , and $233 \mathrm{~L} / \mathrm{mol}$, respectively; (2) the dissolution rate enhancement in the $99 \%$ SLS was $10 \%$ greater than that in the $95 \%$ SLS and $95 \%$ with $0.15 \mathrm{M} \mathrm{NaCl}$ solutions, which were not significantly different; (3) the diffusion coefficients of the drug-loaded micelles estimated from the dissolution experiments were $8.4 \times 10^{-7} \mathrm{~cm}^{2} / \mathrm{s}$ for the $99 \%$ SLS, 9.5 $\times 10^{-7} \mathrm{~cm}^{2} / \mathrm{s}$ for the $95 \% \mathrm{SLS}$, and $1.2 \times 10^{-6} \mathrm{~cm}^{2} / \mathrm{s}$ for the $95 \%$ with $0.15 \mathrm{M} \mathrm{NaCl}$; and (4) the critical micelle concentrations for the $99 \% \mathrm{SLS}$, $95 \%$ SLS, and $95 \%$ SLS with $0.15 \mathrm{M} \mathrm{NaCl}$ were $6.8,4.2$, and $0.35 \mathrm{mM}$, respectively. The results of this study clearly illustrate the sensitivity of the micelle to impurities and electrolytes with regard to size and loading capacity and the effect these changes have on the solubility and dissolution rate. Therefore, when using surfactants in dissolution media for in vitro testing of dosage forms, consideration must be given to the level of impurities present so that the results are consistent and reliable. Intrinsic dissolution rate, surface tension, or solubility measurements may be useful, convenient methods for identifying changes in the surfactant due to either degradation or lot-to-lot variability.
\end{abstract}

\section{Introduction}

The use of surfactants over other dissolution media for in vitro dissolution testing of water-insol uble drugs has increased in recent years because of the mechanistic similarities to in vivo dissolution. ${ }^{1}$ Sodium lauryl sulfate (SLS), a surfaceactive agent commonly used for this purpose, can be purchased in several grades with the increase in material cost directly proportional to the purity ${ }^{2}$. Studies have shown that for SLS, lauryl alcohol impurities produce significant changes in the surface tension-log concentration profile, such as minimums and curve shifts, but little work has been done to correlate surfactant purity to changes in the dissolution rate of water-

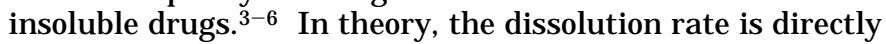
proportional to the diffusivity of the drug-loaded micelle, and any change in the formation of the micelle can have an effect. ${ }^{7-11}$ However, flux is also a function of the solubility of the drug molecule into the micelle, and the outcome may not be predictable based on the micelle diffusivity alone. Therefore, the objective of this research is to measure the solubility and intrinsic dissolution rate of carbamazepine (CBZ) in 99\% pure SLS, $95 \%$ pure SLS, and 95\% pure SLS in $0.15 \mathrm{M} \mathrm{NaCl}$

${ }^{\otimes}$ Abstract published in Advance ACS Abstracts, December 1, 1996. to determine the effect of surface active impurities, such as lauryl alcohol.

\section{Theoretical Section}

Micelle-facilitated dissolution of solids involves both solubilization of the drug into the micelle and transport of the drug-loaded micelle from the solid. The solubilization step has been described as an equilibrium between the solute in the aqueous phase and the micelle as follows ${ }^{7}$ (see Glossary for notations and definitions):

$$
\left[C_{s}\right]+\left[C_{m}\right] \stackrel{k^{*}}{\rightarrow}\left[C_{s m}\right]
$$

where $C_{s}$ is the aqueous concentration of the drug, $C_{m}$ is the concentration of the micelle, and $\mathrm{C}_{\mathrm{sm}}$ is the concentration of the drugloaded micelle, and the equilibrium coefficient, $k^{*}$, is defined as follows:

$$
\mathrm{k}^{*}=\frac{\left[\mathrm{C}_{\mathrm{sm}}\right]}{\left[\mathrm{C}_{\mathrm{s}}\right]\left[\mathrm{C}_{\mathrm{m}}\right]}
$$

At steady state, the convective-diffusion transport of the solute and micelle-solubilized solute is given by eq $2: 12$

$$
0=D_{i} \frac{d^{2} C_{i}}{d z^{2}}-v_{z} \frac{d C_{i}}{d z}
$$

where $D_{i}$ is the diffusion coefficient, $v_{i}$ is the fluid velocity and $\mathrm{i}$ corresponds to the micelle, solute, or drug-loaded micelle. If the fluid velocity is known, as in the case of the well-defined hydrodynamics of the rotating disk, then eq 2 can be solved to eq $3: 10,13,14$

$$
J_{\text {total }}=0.62 \mathrm{D}_{\text {eff }}^{2 / 3} \omega^{1 / 2} v^{-1 / 6} \mathrm{C}_{\text {total }}
$$

where $\mathrm{J}$ total is the total flux, $\mathrm{C}_{\text {total }}$ is the total amount of drug in solution, $\omega$ is the rotational speed of the disk, $v$ is the kinematic viscosity, and $D_{\text {eff }}$ is the effective diffusivity. The effective diffusivity is defined as: ${ }^{15}$

$$
D_{\text {eff }}^{2 / 3}=\frac{D_{s}^{2 / 3}+k^{*}\left[C_{m}\right] D_{s m}^{2 / 3}}{1+k^{*}\left[C_{m}\right]}
$$

where $D_{s}$ is the diffusivity of the solute and $D_{s m}$ is the diffusivity of the drug-loaded micelle.15 The reaction factor (i.e., the total flux divided by the flux of the solute) is given by eq $5:{ }^{15}$

$$
\phi=\frac{J_{\text {total }}}{J_{\text {solute }}}=1+\frac{D_{\text {sm }}^{2 / 3}}{D_{\mathrm{s}}^{2 / 3}} k^{2}\left[C_{m}\right]
$$

Equation 5 shows that the flux enhancement is a function of the diffusivity of the drug-loaded micelle and the degree of solubilization of the drug in the micelle.

The solubilizing effect of surfactants can be expressed as the total solubility of the drug in the surfactant solution divided by the aqueous solubility as in eq 6: ${ }^{7}$ 


$$
\frac{\mathrm{S}_{\text {total }}}{\mathrm{S}_{\text {water }}}=1+\mathrm{k}^{*}\left[\mathrm{C}_{\mathrm{m}}\right]
$$

where $\mathrm{S}_{\text {total }}$ is the total solubility and $\mathrm{S}_{\text {water }}$ is the aqueous concentration of the solute. ${ }^{8}$ Equation 6 allows for easy calculation of the equilibrium coefficient by simply taking the slope of a line regressed through a plot of the solubility enhancement as a function of the surfactant concentration. Changes in the solubility of the drug in the micelle will be reflected as a change in the equilibrium coefficient.

\section{Experimental Section}

Materials and Data Analysis-Anhydrous (CBZ) and 99\% SLS were purchased from Sigma Chemical Company (St. Louis, MO). The 95\% SLS was purchased from Fisher-Scientific (Pittsburgh, PA). The CBZ dihydrate was prepared by recrystallization of anhydrous CBZ with water. The presence of the dihydrate was verified by differential scanning calorimetry (DSC), thermogravimetric analysis (TGA), and Karl Fischer analysis. ${ }^{19,20}$ Distilled, deionized filtered water was used for all solubility and dissolution experiments. The rotating disk was constructed of stainless steel, with a tablet radius of $0.55 \mathrm{~cm}$ and an overall disk radius of $2.50 \mathrm{~cm} .{ }^{21}$ Statistical analysis, model fitting, and parameter estimations were performed with SYSTAT Statistics Software (SYSTAT, Intelligent Software, Evanston, IL).

Dissolution Experiments-To determine the intrinsic dissolution rate, CBZ powder was compressed in a rotating disk die at $9 \times 10^{7}$ $\mathrm{Pa}$ for 20 min (Fred S. Carver, Inc., Summit, NJ). The dissolution experiments were performed at $310 \pm 1 \mathrm{~K}$ in a jacketed beaker that was heated by a water bath to control the temperature, and sink conditions were maintained throughout the entire experiment. The dissolution media from the rotating disk were continuously circulated through the ultraviolet (UV) spectrophotometer flow cells with a peristaltic pump (Masterflex, Cole-Parmer Scientific, Niles, IL), and the UV absorbance was recorded at 2-min intervals (Perkin EImer Lambda 3B UVNIS Spectrophotometer). A contact digital tachometer (Cole-Parmer Scientific, Niles, IL) was used to monitor the rotational speed of the disk. Rapid conversion of anhydrous CBZ to the dihydrate upon exposure to the dissolution media was assumed. ${ }^{19}$ The rotating disk-dissolution experiments were run at 50, 100, 200, and $300 \mathrm{rpm}$ in triplicate except for the $1 \%$ SLS in $0.15 \mathrm{M} \mathrm{NaCl}$ at 50 rpm which was performed in duplicate.

The SLS solutions were prepared the day of the experiment and used within $4 \mathrm{~h}$ of preparation. Prior to the start of the experiment, the $99 \%$ SLS was further purified by foaming the solution (shaking vigorously) and then discarding the foam. ${ }^{22}$ This process was repeated three times. Although this procedure does not remove all of the lauryl alcohol impurities, it represents a practical method of decreasing the level of surface-active impurities in the solution.

Solubility Experiments-The solubility of CBZ dihydrate in different concentrations of surfactant was determined by placing excess CBZ in 20-mL scintillation vials containing solution and gently agitating the vials on an orbital shaker-water bath maintained at $310 \pm 1 \mathrm{~K}$. Samples were removed at 29 and $49 \mathrm{~h}$ and filtered, and CBZ concentrations were measured at $285 \mathrm{~nm}$ (Perkin Elmer Lambda 3B UVNIS spectrophotometer). ${ }^{24}$ No significant change in concentration between the samples was observed. The solubility experiments were performed in triplicate and are reported as the mean \pm standard of the mean.

Viscosity-Assuming a dilute system (i.e., no micelle-micelle interactions), the viscosity of the fluid surrounding the transporting species was taken to be that of water at $310 \pm 1 \mathrm{~K}$, and a value of $0.007 \mathrm{~cm}^{2} / \mathrm{s}$ (and 0.007 poise) was used for the viscosity term in all calculations. To verify this assumption experimentally, the kinematic viscosity was measured for all surfactant concentrations studied and was found to range from 0.007 stokes for water to 0.0076 stokes for $2 \%$ SLS. When taken to the $1 / 6$ power, these values become 0.443 for the latter and 0.437 for the former, resulting in at most a $1 \%$ increase in kinematic viscosity $2 \%$ SLS over that in water. The conversion of the kinematic viscosity to poise was performed with the following equation, assuming the density of water to be $0.993 \mathrm{gm} /$ $\mathrm{cm}^{3}$ at $37^{\circ} \mathrm{C}: 8 v=\eta / \rho$

Surface Tension Measurements-The surface tension experiments were performed in triplicate at $310 \pm 1 \mathrm{~K}$ by the Wilhelmy Plate method (Rosano Surface Tensiometer, Laboratory Products, Inc.,

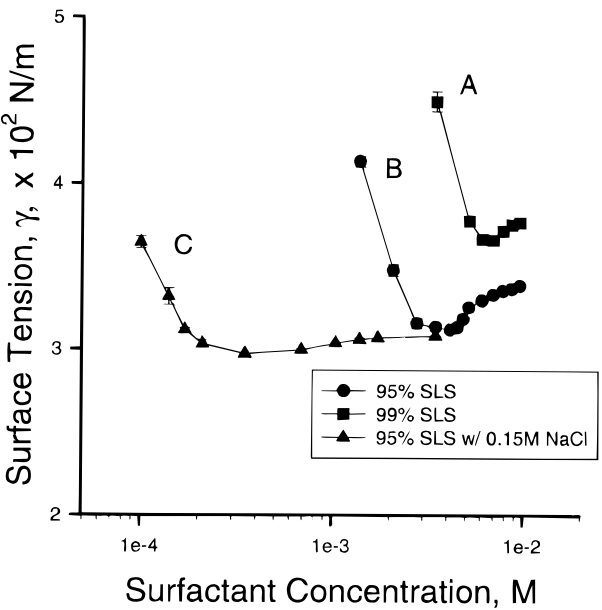

Figure 1-Surface tension versus the log of the surfactant concentration for $99 \%$ SLS, $95 \%$ SLS, and $95 \%$ SLS with $0.15 \mathrm{M} \mathrm{NaCl}$.

Boston, MA). The platinum plate was cleaned between each measurement by heating to "red hot" with a butane flame.

The critical micelle concentration (CMC) of each solution was determined by first plotting the surface tension of the solution as a function of the log of the concentration of the surfactant. A perpendicular line was drawn from the minimum of the curve to the x-axis, and the CMC was read from the point of intersection. ${ }^{3}$

\section{Results and Discussion}

Surface Tension Measurements and Critical Micelle Calculation-Figure 1 is a plot of the surface tension versus the log of the surfactant concentration for $99 \%$ pure SLS in water, $95 \%$ pure SLS in water, and $95 \%$ pure SLS in $0.15 \mathrm{M}$ $\mathrm{NaCl}$. The surface tension profiles of the three solutions showed significant differences. First, at pre-micelle concentrations, the slopes of the $99 \%$ and $95 \%$ SLS curves (curves A and $B$, respectively) are more negative $(-0.036$ and $0.033 \mathrm{~N} / \mathrm{m} /$ $\mathrm{M}$, respectively) than the slope of the $95 \%$ SLS with $0.15 \mathrm{M}$ $\mathrm{NaCl}$ (curve C, $-0.023 \mathrm{~N} / \mathrm{m} / \mathrm{M}$ ). This difference is likely due to a greater concentration of surface-active impurities present in the $95 \%$ and $99 \%$ SLS solutions compared with the detergent monomers. In the case of the 95\% SLS with 0.15 $\mathrm{M} \mathrm{NaCl}$, charge screening from the $\mathrm{NaCl}$ at the interface allows for a greater concentration of surfactant molecules while suppressing the effect of the alcohol impurity. ${ }^{3}$

Second, as the concentration of the surfactant increases, micelles form and embody the impurity, thereby reducing the bulk and surface concentration of the alcohol impurity and producing the observed minimum. 3,19 The level of involvement of the alcohol impurity in the formation of mixed micelles significantly decreases, as does the surface activity of the nonelectrolyte impurity, upon addition of sodium chloride. The reduction of surface-active impurities at the interface is observed as a decrease in the minimum for the $95 \%$ SLS with $0.15 \mathrm{M} \mathrm{NaCl}^{3}$

Finally, the CMCs of the three solutions (99\% SLS, 95\% SLS, and $95 \%$ SLS in $0.15 \mathrm{M} \mathrm{NaCl}$ ), estimated from the surface tension curves, are $0.35,4.2$, and $6.8 \mathrm{mM}$, respectively. Although these differences just discussed are not extraordinary and have been described elsewhere, they do verify the presence of highly surface-active lauryl alcohol molecules at pre-CMC concentrations followed by the formation of mixed micelles at higher surfactant concentrations. ${ }^{3}$

Solubility Enhancement-The solubility enhancement of CBZ in the 99\% SLS, 95\% SLS, and 95\% SLS with $0.15 \mathrm{M}$ $\mathrm{NaCl}$ solutions is shown in Figure 2. The equilibrium coefficients were determined by regression analysis with eq 


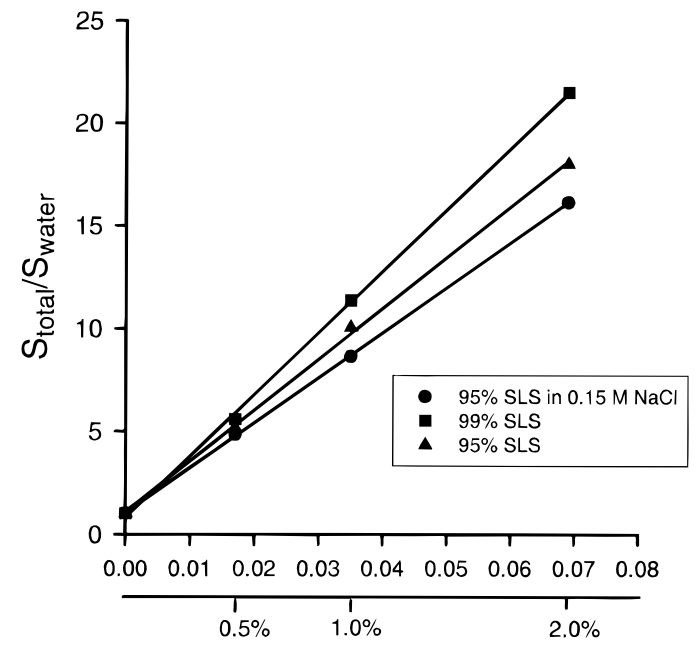

SLS Concentration, $\mathrm{M} / \% \mathrm{w} / \mathrm{V}$

Figure 2-Solubility enhancement of CBZ in aqueous solutions of $99 \%$ SLS, $95 \%$ SLS, and $95 \%$ SLS with $0.15 \mathrm{M} \mathrm{NaCl}$.

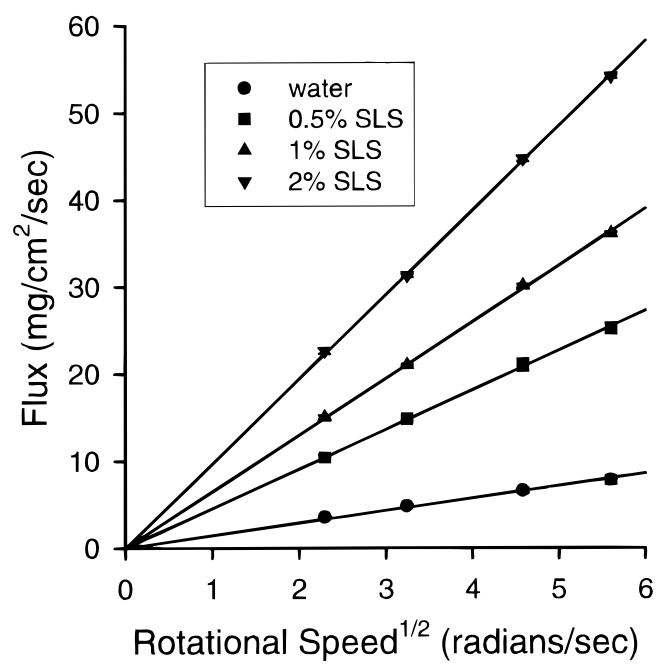

Figure 3-Intrinsic dissolution rate of CBZ in $99 \%$ SLS $(n=3)$.

5 and are 295,265 , and $233 \mathrm{~L} / \mathrm{M}$, respectively. The differences in the solubility enhancement and equilibrium coefficient of CBZ in the three solutions are significant and may be due to competition between the solute and lauryl alcohol monomer for the micelle, closeness of packing between the monomers in the micelle as a result of charge screening from the electrolyte, or salting-out effects. ${ }^{13,19,26}$ For example, the repulsion between monomers due to the charge on the surfactant molecule may allow drug to be easily incorporated in the micelle. The presence of electrolytes, resulting in charge screening, may reduce this separation, thereby decreasing the solubility. In addition, surface-active impurities, such as lauryl alcohol, may lead to less space available for other molecules, resulting in a decrease in the solubility as observed.

Intrinsic Dissolution Rate from a Rotating Disk and Dissolution Rate Enhancement-The intrinsic dissolution rates of CBZ in the three SLS solutions as a function of rotational speed and SLS concentration are shown in Figures 3,4 , and 5. Based on the observed linear relationship and zero intercept, the dissolution was assumed to be diffusion controlled according to eq 3. The data reported are the average of three experiments \pm the standard error of the

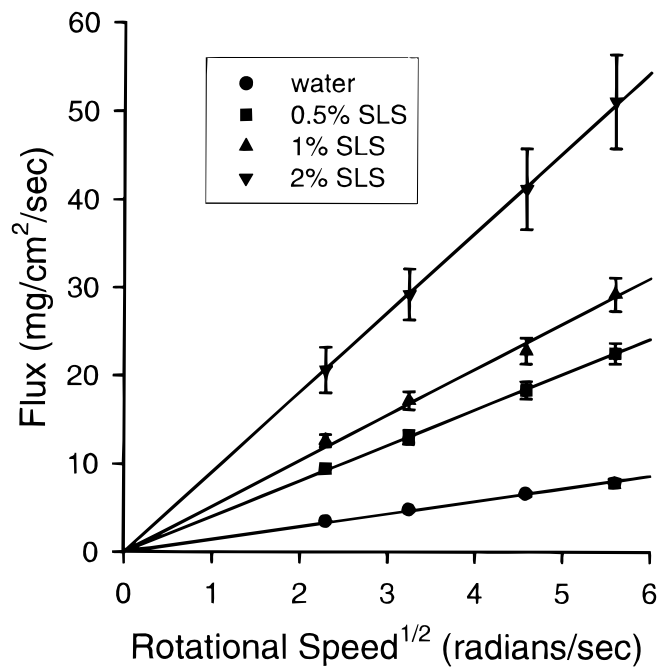

Figure 4-Intrinsic dissolution rate of CBZ in $95 \%$ SLS $(n=3)$.

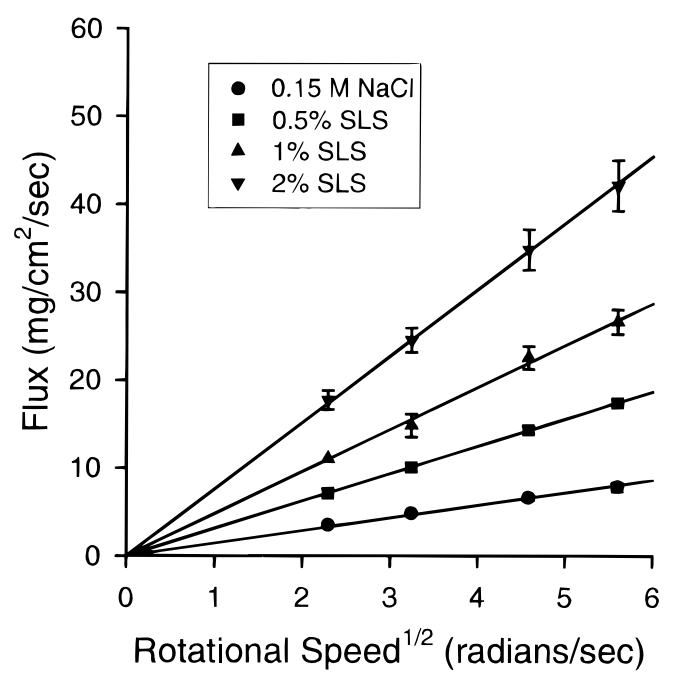

Figure 5-Intrinsic dissolution rate of CBZ in $95 \%$ SLS with $0.15 \mathrm{M} \mathrm{NaCl}(n=$ 3).

mean, except for the 1\% SLS at $50 \mathrm{rpm}$ data point in Figure 5 , which is the average of two experiments.

In Figure 6, the reaction factor (i.e., enhanced dissolution rate) is plotted as a function of surfactant concentration. ${ }^{15}$ The slopes of these lines, as determined by linear regression, are $67.8 \pm 2.8$ for $99 \%$ SLS, $66.4 \pm 3.1$ for $95 \%$ SLS, and $69.3 \pm$ 6.9 for $95 \% \mathrm{SLS}$ with $0.15 \mathrm{M} \mathrm{NaCl}$. Although the slopes are statistically the same, the degree of enhancement of the $99 \%$ pure SLS is $\sim 10 \%$ greater than the other two solutions. All of the regressed lines have intercepts greater than the theoretical value of one that is likely due to the model assumption that all micelles formed are the same size. Differences in the degree of contribution to the total flux from various sized micelles can result in deviations from theory and have been discussed elsewhere. ${ }^{15}$

A positive deviation of the experimentally determined effective diffusivity from the theoretical effective diffusivity is also observed in Figure 7. The effective diffusivities in this figure were calculated from the slopes of the intrinsic dissolution rate data in Figures 3-5 with eq 3, the theoretical curve (dotted line) was calculated with eq 4, and the equilibrium coefficient and the micellar diffusivity were based on the $99 \%$ SLS data. In contrast, note that the diffusivity term is not present in the equation for solubility enhancement (eq 6) and an intercept of one is observed in Figure 2. 


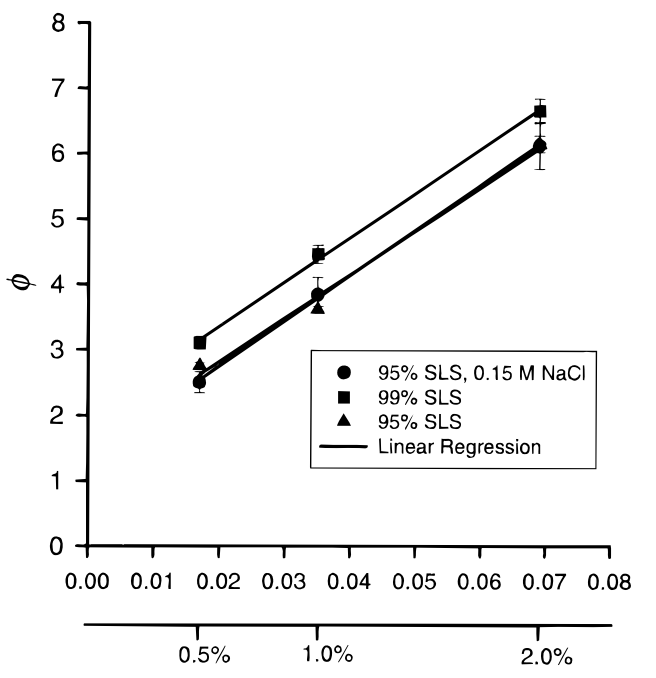

SLS Concentration, $\mathrm{M} / \% \mathrm{w} / \mathrm{v}$

Figure 6-Reaction factor for the intrinsic dissolution rate of $\mathrm{CBZ}$ in aqueous solutions of $99 \%$ SLS, $95 \%$ SLS, and $95 \%$ SLS with $0.15 \mathrm{M} \mathrm{NaCl}$.

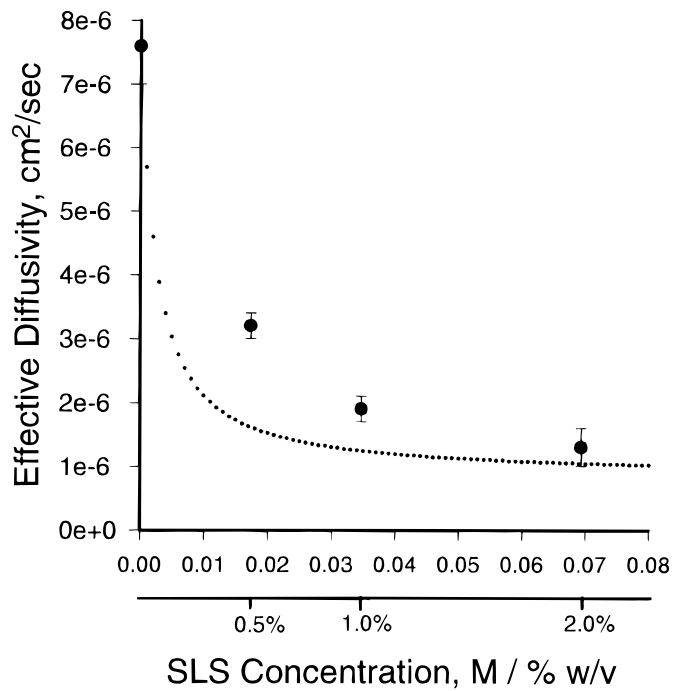

Figure 7-Effective diffusivity versus surfactant concentration. The symbols are determined from the intrinsic dissolution rate, and the dotted line is estimated from eqs 4 and 5 .

The diffusion coefficients of the drug-loaded micelles were estimated from linear regression of the reaction factor data in Figure 6 and eq 5. The diffusivity of the drug-loaded micelle for the $99 \%, 95 \%$, and $95 \%$ with $0.15 \mathrm{M} \mathrm{NaCl}$ solutions are $8.4 \times 10^{-7}, 9.5 \times 10^{-7}$, and $1.2 \times 10^{-6} \mathrm{~cm}^{2} / \mathrm{s}$, respectively. These values are within the range of micelle diffusion coefficients reported in the literature for SDS in the presence of impurities and electrolytes determined by other methods. ${ }^{24-26}$ The differences in micelle size observed for the three solutions can be attributed to several factors, such as, charge repulsion between the hydrophilic groups of the SLS monomer or the incorporation of alcohol impurities forming mixed micelles. Addition of alcohol impurities or el ectrolytes may also reduce this repulsion by producing micelles that are more closely packed.

Effect of Surfactant Purity and Electrolytes on In Vitro Dissolution Media for Product Testing-The results of this study clearly illustrate the sensitivity of the micelle to impurities and electrolytes with regard to size and loading capacity. The differences observed in the intrinsic dissolution profiles for the three solutions strongly suggest a potential for failure in meeting product in vitro dissolution test specifications based on variability that is unrelated to formulation or processing. For example, USP/NF purity specifications only require that SLS be between 96 and 100\%. However, the data in Figure 6 show a 10\% difference between reaction factors for the $99 \% \mathrm{SLS}$ and $95 \% \mathrm{SLS}$ or $95 \% \mathrm{SLS}$ with $0.15 \mathrm{M} \mathrm{NaCl}$; so, it is reasonable to expect that dissolution profiles for solutions prepared with SLS within this range of purity may also exhibit significant differences. ${ }^{28}$ The addition of sodium chloride to the $95 \%$ SLS did not effect the dissolution rate enhancement, but it did decrease the solubility enhancement and reduce the minimum in the log concentration-surface tension plots. However, a general statement for all waterinsoluble drugs regarding the effect of el ectrolytes on dissolution cannot be made without more data. Each system must be considered individually to take into account charge screening and salting out effects on the total flux, especially because SLS is not the only surfactant available for this use.

When using surfactants in dissolution media for in vitro testing of dosage forms, consideration must be given to the level of impurities present so that the results are consistent and reliable. Experimental methods for measuring or identifying the presence of surface-active impurities may be necessary to insure reliable in vitro dissolution results. Such experiments should provide measurement of physicochemical properties of the monomer and micelle that have an impact on the formation, solubilization, and dissolution characteristics of micelles and may include solubility and surface tension measurements. Intrinsic dissolution testing may be useful for determining the combined effect that impurities and electrolyte have on the total flux. When analyzed in conjunction with the dissolution profile of the dosage form, the intrinsic dissolution rate can be used to help identify other problems intrinsic to water-insoluble drugs such as particle aggregation.

\section{Glossary}

$\mathrm{C}_{i}$, concentration of species $\mathrm{i}\left(\mathrm{mg} / \mathrm{cm}^{3}\right) ; \mathrm{C}_{i(0)}$, concentration of species $i$ at the surface of the solid $\left(\mathrm{mg} / \mathrm{cm}^{3}\right) ; C_{i(b)}$, concentration of species $i$ in the bulk $\left(\mathrm{mg} / \mathrm{cm}^{3}\right)$; $D_{i}$, diffusion coefficient of species i $\left(\mathrm{cm}^{2} / \mathrm{s}\right) ; j$, flux $\left(\mathrm{mg} / \mathrm{cm}^{2} / \mathrm{s}\right)$; $\mathrm{k}^{*}$, equilibrium coefficient $\left(\mathrm{cm}^{3} / \mathrm{mg}\right) ; S_{i}$, solubility of species $i$ $\left(\mathrm{mg} / \mathrm{cm}^{3}\right) ; \gamma_{i}$, surface tension $(\mathrm{N} / \mathrm{m}) ; v$, kinematic viscosity $\left(\mathrm{cm}^{2} / \mathrm{s}\right) ; \omega$, rotational speed (radians/s); $v_{\mathrm{z}}$, axial velocity $-0.51 \cdot(\sqrt{\omega / v}) \cdot z^{2}$

\section{References and Notes}

1. Shah, V. P.; Konecny, J . J .; Everett, R. L.; McCullough, B.; Noorizadeh, A. C.; Skelly, J . P. Pharm. Res. 1989, 6, 612-618.

2. Sigma Chemical Company, 1994.

3. Weiner, N. D.; Flynn, G. L. 1974, 22, 2480-2483.

4. Miles, G. D. J. Phys. Chem. 1945, 49, 71-76.

5. Harrold, S. P. J . Phys. Chem. 1959, 63, 317-318.

6. Elworthy, P. H.; Mysels, K. J . J . Colloid Interface Sci. 1966, 21, 331-347.

7. Higuchi, W. I. J . Pharm. Sci. 1964, 53, 532-535.

8. Amidon, G. E.; Higuchi, W. I.; Ho, N. F. H.J . Pharm. Sci. 1982, $71,77-84$.

9. DeSmidt, J . H.; Offringa, J . C. A.; Crommelin, D. J . A. J . Pharm. Sci. 1987, 76, 711-714.

10. Gibaldi, M.; Feldman, S.; Wynn, R.; Weiner, N. D. J . Pharm. Sci. 1968, 57, 787-791.

11. Singh, P.; Desai, S. J .; Flanagan, D. R.; Simonelli, A. P.; Higuchi, W. I. J . Pharm. Sci. 1968, 6, 959-965.

12. Bird, R. B.; Stewart, W. E.; Lightfoot, E. N. Transport Phenomena; J ohn Wiley \& Sons: New York, NY, 1960.

13. Cochran, W. G. Proc. Cambridge Phil. Soc. 1934, 30, 365-375.

14. Levich, V. G. In Physico-Chemical Hydrodynamics; PrenticeHall: Englewood Cliffs, NJ , 1962; pp 39-72. 
15. Crison, J. R.; Skelly, J. P.; Shah, V. P.; Amidon; G. L. J . Pharm. Sci. 1995, 85, 1005-1011.

16. Krahn, F. U.; Mielck, J. B. Pharm. Acta Helv. 1987, 62, 247254.

17. Lowes, M. M.J .; Caira, M. R.; Lötter, A. P.; VanDerWatt, J . G. J. Pharm. Sci. 1987, 76, 744-752.

18. Wood, J. H.; Syarto, J. E.; Letterman, H. J . Pharm. Sci. 1965, 54, 1068.

19. Brady, A. P. J . Phys. Colloid Chem. 1949, 53, 56-66.

20. Weast, R. C. Handbook of Chemistry and Physics; 54th ed.; CRC: Cleveland, $\mathrm{OH}, 1973-74 ; \mathrm{pp} F-45$

21. Aboul-E nein, H. Y.; Al-Badr, A. A. Carbamazepine. In Analytical Properties of Drug Substances; Flory, X., Ed.; Academic: 1980; pp 87.

22. Reid, R. C.; Prausnitz, J . M.; Sherwood, T. K. The Properties of Gases and Liquids, 3rd ed.; McGraw-Hill Book: New York, NY, 1977; p 573.
23. Mazer, N. A.; Benedek, G. B.; Carey, M. C. J . Phys. Chem. 1976, 80, 1075-1084.

24. Almgren, M.; Lofroth, J . E. J . Colloid Interface Sci. 1981, 81, 486-499.

25. Weinheimer, R. M.; Evans, D. F.; Cussler, E. L. J . Colloid Interface Sci. 1981, 80, 357.

26. Preston, W. C. J . Phys. Colloid Chem. 1948, 52, 84-97.

27. Klevens, H. B. J . Am. Chem. Soc. 1950, 72, 3780-3785.

28. USP/ NF, United States Pharmacopeial Convention, Inc., Rand McNally: Taunton, MA, 1995.

\section{Acknowledgments}

This research was partially funded by Grant FD01462 from the Food and Drug Administration.

J S960105T 